\title{
Equation of state and thickness of the inner crust of neutron stars
}

\author{
Fabrizio Grill, Helena Pais, Constança Providência, and Isaac Vidaña \\ Centro de Física Computacional, Department of Physics, University of Coimbra, P-3004-516 Coimbra, Portugal
}

Sidney S. Avancini

Depto de Física - CFM - Universidade Federal de Santa Catarina Florianópolis - SC - CP. 476 - CEP 88.040 - 900 - Brazil

(Received 17 January 2014; revised manuscript received 10 September 2014; published 17 October 2014)

\begin{abstract}
The cell structure of $\beta$-stable clusters in the inner crust of cold and warm neutron stars is studied within the Thomas-Fermi approach by using relativistic mean-field nuclear models. The relative size of the inner crust and the pasta phase of neutron stars is calculated, and the effect of the symmetry energy slope parameter $L$ on the profile of the neutron star crust is discussed. It is shown that, while the size of the total crust is mainly determined by the incompressibility modulus, the relative size of the inner crust depends on $L$. It is found that the inner crust represents a larger fraction of the total crust for smaller values of $L$. Finally, it is shown that, at finite temperature the pasta phase in $\beta$-equilibrium matter essentially melts above 5 to $6 \mathrm{MeV}$, and the onset density of the rod-like and slab-like structures does not depend on the temperature.
\end{abstract}

DOI: 10.1103/PhysRevC.90.045803

\section{INTRODUCTION}

Nowadays, the existence of the so-called pasta phase [1-10] in the inner crust of a neutron star close to the crust-core transition is generally accepted. Constituted by several types of complex structures such as, e.g., rods and slabs, the pasta phase is a frustrated system formed as a result of the competition between the strong and the electromagnetic interactions.

The effect of the density dependence of the symmetry energy on the pasta phase has been discussed in several works [11-14]. In particular, it has been shown that, for very large values of the symmetry energy slope parameter $L=3 n_{0}\left[\partial E_{\text {sym }}(n) / \partial n\right]_{n_{0}}$, nonspherical structures (e.g., rod like or slab like) are not expected in $\beta$-equilibrium matter, and that the number of nucleons in the clusters as well as the cluster proton fraction and the size of the Wigner-Seitz (WS) cell are sensitive to this quantity. It has been also discussed that $L$ may have quite dramatic effects on the cell structure if its value is very large or small $[9,12,13]$.

One of the present issues in the study of neutron stars is the determination of its crust properties, which is possible from the observations of crust cooling [15]. Understanding the crust properties is essential since it acts as a filter for any information coming from the neutron star core. Since the timescale of cooling of the neutron star crust is proportional to the square of the crust thickness [16], the calculation of this quantity may allow us to set constraints on the equation of state (EoS) of the crust. Also in the determination of the crustal moment of inertia, an essential quantity to interpret pulsar glitches [16], the thickness of the crust plays an important role. In particular, it has been recently discussed whether the crustal moment of inertia is large enough to describe the pulsar glitches [17-19].

The cooling of proto-neutron stars is mostly driven by neutrino diffusion during the first seconds. The existence of nonhomogeneous matter will affect the evolution of a supernova or proto-neutron star matter; in particular, the diffusion of neutrinos out of the star [3,20-22]. A phenomena such as critical opalescence may occur at the melting temperature during the cooling process due to the trapping of neutrinos. In
PACS number(s): 21.65.Cd, 24.10.Jv, 26.60.-c, 97.60.Jd

Ref. [21] it was shown that nonspherical pasta phases decrease the opacity at low energies and, at finite temperature, the static structure factor that defines the neutrino cross section decreases, leading to a faster cooling.

In the present work the effect of $L$ on the size of the inner crust will be discussed within a Thomas-Fermi (TF) formalism in the WS approximation in the framework of relativistic mean-field (RMF) nuclear models [6-9]. The Tolman-Oppenheimer-Volkov equations (TOV) [23] will be integrated and the size of the inner crust as well as the localization of the pasta structures identified. In particular, it is shown that smaller values of $L$ favor a wider slab phase and a larger relative size of the inner crust with respect to the total crust, and a steeper crust profile.

We also study the effect of temperature on the size of the inner crust. It is shown that pasta clusters in $\beta$ equilibrium completely melt for temperatures above 5 to $6 \mathrm{MeV}$. These results agree partially with the predictions obtained within a dynamical spinodal (DS) approach [24-26]. A similar calculation was done with Skyrme forces in Ref. [27]. The melting of the pasta phase was previously studied in Refs. [28,29], where the effect of thermal fluctuations was taken into account. It is expected that, if thermal fluctuations are larger than the radius of the WS cell, the WS approximation breaks down. However, thermal fluctuations are not considered in the present calculation and, therefore, the reader must interpret our results just as upper limits.

Some preliminary results of the present study have been published in Ref. [14].

The paper is organized as follows: The formalism is briefly reviewed in Sec. II. Section III is devoted to the presentation and discussion of the results while the main conclusions are given in Sec. IV.

\section{FORMALISM}

To describe the inner crust at zero and finite temperatures we apply the self-consistent TF formalism presented in 
Refs. [7-9]. We recall that the description of hot nuclei within a generalization of the liquid-drop model has already been applied in the framework of the statistical formulation of the multifragmentation of finite nuclei with nonrelativistic nuclear models in the mid eighties [30]. We use relativistic meanfield nuclear models with constant couplings and nonlinear terms [31], and with density-dependent couplings [32]. Within the first class of models, which we designate by nonlinear Walecka models, we consider the following ones: NL3 [33] with nonlinear $\sigma$ terms, TM1 [34] with nonlinear $\sigma$ and $\omega$ terms, NL3 $3_{\omega \rho}$ including also nonlinear $\omega \rho$ terms which allow the modulation of the density dependence of the symmetry energy [35], FSU [36], and IU-FSU [37] with nonlinear $\sigma$, $\omega$, and $\omega \rho$ terms. NL3 and FSU have been chosen because they are frequently applied in the description of nuclear matter and stellar hadronic matter; see, e.g., Refs. [18,38,39]. The results obtained with them have to be taken into account according to their limitations: NL3 is too hard at high densities and has a too large symmetry energy slope, and FSU is too soft and does not describe a $2 M_{\odot}$ neutron star; however, we expect it describes well the crust EoS. Within the second class of models with density-dependent couplings we consider the models DD-ME2 [40] and DD-ME $\delta$ [41]. The latter, among the parametrizations considered, is the only one including the $\delta$ meson.

All the equations that allow the performance of the TF calculation are derived from the Lagrangian density

$$
\mathcal{L}=\sum_{i=n, p} \mathcal{L}_{i}+\mathcal{L}_{e}+\mathcal{L}_{\sigma}+\mathcal{L}_{\omega}+\mathcal{L}_{\rho}+\mathcal{L}_{\delta}+\mathcal{L}_{\gamma}+\mathcal{L}_{n l},
$$

where the nucleon Lagrangian reads

$$
\mathcal{L}_{i}=\bar{\psi}_{i}\left[\gamma_{\mu} i D^{\mu}-M^{*}\right] \psi_{i}
$$

with

$$
\begin{aligned}
i D^{\mu} & =i \partial^{\mu}-\Gamma_{\omega} \omega^{\mu}-\frac{\Gamma_{\rho}}{2} \boldsymbol{\tau} \cdot \rho^{\mu}-e \frac{1+\tau_{3}}{2} A^{\mu}, \\
M^{*} & =M-\Gamma_{\sigma} \sigma-\Gamma_{\delta} \boldsymbol{\tau} \cdot \boldsymbol{\delta},
\end{aligned}
$$

and the electron Lagrangian is given by

$$
\mathcal{L}_{e}=\bar{\psi}_{e}\left[\gamma_{\mu}\left(i \partial^{\mu}+e A^{\mu}\right)-m_{e}\right] \psi_{e} .
$$

The meson and electromagnetic Lagrangian densities are

$$
\begin{aligned}
\mathcal{L}_{\sigma}= & \frac{1}{2}\left(\partial_{\mu} \sigma \partial^{\mu} \sigma-m_{\sigma}^{2} \sigma^{2}\right), \\
\mathcal{L}_{\omega}= & \frac{1}{2}\left(-\frac{1}{2} \Omega_{\mu \nu} \Omega^{\mu \nu}+m_{\omega}^{2} \omega_{\mu} \omega^{\mu}\right), \\
\mathcal{L}_{\rho}= & \frac{1}{2}\left(-\frac{1}{2} \mathbf{R}_{\mu \nu} \cdot \mathbf{R}^{\mu \nu}+m_{\rho}^{2} \boldsymbol{\rho}_{\mu} \cdot \boldsymbol{\rho}^{\mu}\right), \\
\mathcal{L}_{\delta}= & \frac{1}{2}\left(\partial_{\mu} \boldsymbol{\delta} \partial^{\mu} \boldsymbol{\delta}-m_{\delta}^{2} \boldsymbol{\delta}^{2}\right), \\
\mathcal{L}_{\gamma}= & -\frac{1}{4} F_{\mu \nu} F^{\mu \nu}, \\
\mathcal{L}_{n l}= & -\frac{1}{3 !} \kappa \sigma^{3}-\frac{1}{4 !} \lambda \sigma^{4}+\frac{1}{4 !} \xi \Gamma_{\omega}^{4}\left(\omega_{\mu} \omega^{\mu}\right)^{2} \\
& +\Lambda_{\omega} \Gamma_{\omega}^{2} \Gamma_{\rho}^{2} \omega_{\nu} \omega^{\nu} \boldsymbol{\rho}_{\mu} \cdot \boldsymbol{\rho}^{\mu},
\end{aligned}
$$

where $\Omega_{\mu \nu}=\partial_{\mu} \omega_{v}-\partial_{\nu} \omega_{\mu}, \mathbf{R}_{\mu \nu}=\partial_{\mu} \boldsymbol{\rho}_{\nu}-\partial_{\nu} \boldsymbol{\rho}_{\mu}-\Gamma_{\rho}\left(\boldsymbol{\rho}_{\mu} \times\right.$ $\left.\boldsymbol{\rho}_{\nu}\right)$, and $F_{\mu \nu}=\partial_{\mu} A_{\nu}-\partial_{\nu} A_{\mu}$. The four coupling parameters $\Gamma_{\sigma}, \Gamma_{\omega}, \Gamma_{\rho}$, and $\Gamma_{\delta}$ of the mesons to the nucleons are density dependent in the relativistic density-dependent models considered; namely, DD-ME2 [40] and DD-ME $\delta$ [41]. The
TABLE I. Nuclear matter properties at saturation: density $\left(n_{0}\right)$, energy $\left(E_{0}\right)$, incompressibility $\left(K_{0}\right)$, skewness $\left(Q_{0}\right)$, symmetry energy $\left(E_{\text {sym }}\right)$, and symmetry energy slope parameter $(L)$.

\begin{tabular}{lcccrcr}
\hline \hline Model & $\begin{array}{c}n_{0} \\
\left(\mathrm{fm}^{-3}\right)\end{array}$ & $\begin{array}{c}E_{0} \\
(\mathrm{MeV})\end{array}$ & $\begin{array}{c}K_{0} \\
(\mathrm{MeV})\end{array}$ & $\begin{array}{c}Q_{0} \\
(\mathrm{MeV})\end{array}$ & $\begin{array}{c}E_{\text {sym }} \\
(\mathrm{MeV})\end{array}$ & $\begin{array}{c}L \\
(\mathrm{MeV})\end{array}$ \\
\hline NL3 & 0.148 & -16.24 & 270.7 & 203 & 37.3 & 118.3 \\
TM1 & 0.145 & -16.26 & 280.4 & -295 & 36.8 & 110.6 \\
FSU & 0.148 & -16.30 & 230.0 & -523 & 32.6 & 60.5 \\
NL3 $\omega \rho$ & 0.148 & -16.30 & 272.0 & 203 & 31.7 & 55.2 \\
DD-ME $\delta$ & 0.152 & -16.12 & 219.1 & -741 & 32.4 & 52.9 \\
DD-ME2 & 0.152 & -16.14 & 250.8 & 478 & 32.3 & 51.4 \\
IU-FSU & 0.155 & -16.40 & 231.2 & -288 & 31.3 & 47.2 \\
\hline \hline
\end{tabular}

nonlinear term $\mathcal{L}_{n l}$ is absent in these models. In all the other models, NL3 [33], TM1 [34], NL3 ${ }_{\omega \rho}$ [35], FSU [36], and IU-FSU [37], the couplings are constant and at least some of the nonlinear terms of $\mathcal{L}_{n l}$ are included. In the above Lagrangian density $\boldsymbol{\tau}$ is the isospin operator. For reference, we give in Table I the main properties of the above models at saturation density. We will discuss how sensitive the structure of the nonhomogeneous inner crust of a neutron star is to the properties of the equation of state (EoS).

We use the Thomas-Fermi approximation to describe the nonuniform npe matter inside the Wigner-Seitz unit cell, which is taken to be a sphere, a cylinder, or a slab in three, two, and one dimensions [7,9]. In this approximation npe matter is assumed to be locally homogeneous and, at each point, its density is determined by the corresponding local Fermi momenta. In three dimensions (3D) we consider spherical symmetry and in two-dimensional (2D) symmetry we assume axial symmetry around the $z$ axis. In the TF approximation of nonuniform npe matter, fields are assumed to vary slowly so that baryons can be treated as moving in locally constant fields at each point $[6,7]$. The calculation starts from the grand canonical potential density,

$$
\begin{aligned}
\omega & =\omega\left(\left\{f_{i+}\right\},\left\{f_{i-}\right\}, \sigma, \omega_{0}, \rho_{30}, \delta_{0}\right) \\
& =\mathcal{E}-T \mathcal{S}-\sum_{i=n, p, e} \mu_{i} n_{i},
\end{aligned}
$$

where $\left\{f_{i+}\right\}\left(\left\{f_{i-}\right\}\right)$ with $i=n, p, e$ stands for the neutron, proton, and electron positive (negative) energy distributions, and $\mathcal{S}$ and $\mathcal{E}$ are the total entropy and energy densities, respectively [9]. The equations of motion for the meson fields (see, e.g., Ref. [7] for details) follow from the variational conditions

$$
\frac{\delta \Omega}{\delta \sigma(\mathbf{r})}=\frac{\delta \Omega}{\delta \omega_{0}(\mathbf{r})}=\frac{\delta \Omega}{\delta \rho_{30}(\mathbf{r})}=\frac{\delta \Omega}{\delta \delta_{0}(\mathbf{r})}=0,
$$

where $\Omega=\int d^{3} r \omega$. The numerical algorithm for the description of the neutral npe matter at finite temperature is a generalization of the $T=0$ case which is discussed in detail in Refs. [7,9]. The Poisson equation is always solved by using the appropriate Green's function according to the spatial dimension of interest, and the Klein-Gordon equations are solved by expanding the meson fields in a harmonic oscillator basis with one, two, or three dimensions based on the method 
presented in Refs. [7,9]. The interested reader is referred to these works for details of the calculation.

\section{RESULTS}

In this section we present and discuss the results obtained for the different models considered. The section is divided into three parts. In the first part our attention is focused on the sensitivity of the thickness and structure of the inner crust to the properties of the EoS. In the second part we discuss

TABLE II. Central density $\rho_{c}$ and energy density $\mathcal{E}_{c}$, distance to the center of the star at the phase transitions: homogeneous matterslab phase $R_{\mathrm{h}-\mathrm{s}}$, slab-phase-rod-phase $R_{\mathrm{s}-\mathrm{r}}$, rod-phase-droplet-phase $R_{\mathrm{r}-\mathrm{d}}$, droplet-phase-outer-crust $R_{\mathrm{d}-\mathrm{BPS}}$, and radius of a $1 M_{\odot}, 1.44 M_{\odot}$, $1.6 M_{\odot}$ and maximum-mass neutron star for the all the RMF models considered. For NL3 and TM1 there are neither slabs nor rods: the values marked with an asterisk correspond to the homogeneous matter-droplet phase transition.

\begin{tabular}{|c|c|c|c|c|c|c|c|}
\hline $\begin{array}{l}M \\
\left(M_{\odot}\right)\end{array}$ & $\begin{array}{c}\rho_{c} \\
\left(\mathrm{fm}^{-3}\right)\end{array}$ & $\begin{array}{c}\mathcal{E}_{c} \\
\left(\mathrm{fm}^{-4}\right)\end{array}$ & $\begin{array}{l}R_{\mathrm{h}-\mathrm{s}} \\
(\mathrm{km})\end{array}$ & $\begin{array}{r}R_{\mathrm{s}-\mathrm{r}} \\
(\mathrm{km})\end{array}$ & $\begin{array}{c}R_{\mathrm{r}-\mathrm{d}} \\
(\mathrm{km})\end{array}$ & $\begin{array}{c}R_{\mathrm{d}-\mathrm{BPS}} \\
(\mathrm{km})\end{array}$ & $\begin{array}{c}R \\
(\mathrm{~km})\end{array}$ \\
\hline \multicolumn{8}{|c|}{ NL3 } \\
\hline 1.00 & 0.226 & 1.121 & $12.568^{*}$ & & & 13.516 & 14.543 \\
\hline 1.44 & 0.276 & 1.390 & $13.341^{*}$ & & & 13.980 & 14.637 \\
\hline 1.60 & 0.293 & 1.489 & $13.534^{*}$ & & & 14.094 & 14.663 \\
\hline 2.78 & 0.669 & 4.415 & $12.978^{*}$ & & & 13.141 & 13.292 \\
\hline \multicolumn{8}{|c|}{ TM1 } \\
\hline 1.00 & 0.243 & 1.208 & $12.425^{*}$ & & & 13.411 & 14.408 \\
\hline 1.44 & 0.328 & 1.674 & $13.012^{*}$ & & & 13.648 & 14.267 \\
\hline 1.60 & 0.366 & 1.893 & $13.089^{*}$ & & & 13.639 & 14.158 \\
\hline 2.18 & 0.851 & 5.345 & $11.937^{*}$ & & & 12.169 & 12.381 \\
\hline \multicolumn{8}{|c|}{ FSU } \\
\hline 1.00 & 0.353 & 1.774 & 11.081 & 11.146 & 11.230 & 11.940 & 12.818 \\
\hline 1.44 & 0.543 & 2.865 & 11.342 & 11.382 & 11.432 & 11.855 & 12.346 \\
\hline 1.60 & 0.688 & 3.770 & 11.147 & 11.179 & 11.219 & 11.554 & 11.943 \\
\hline 1.73 & 1.151 & 7.047 & 10.320 & 10.342 & 10.370 & 10.600 & 10.866 \\
\hline \multicolumn{8}{|c|}{$\mathrm{NL}_{\omega \rho}$} \\
\hline 1.00 & 0.255 & 1.259 & 11.394 & 11.469 & 11.578 & 12.452 & 13.421 \\
\hline 1.44 & 0.296 & 1.483 & 12.393 & 12.447 & 12.525 & 13.135 & 13.779 \\
\hline 1.60 & 0.312 & 1.574 & 12.666 & 12.714 & 12.783 & 13.325 & 13.891 \\
\hline 2.76 & 0.686 & 4.486 & 12.643 & 12.659 & 12.681 & 12.851 & 13.011 \\
\hline \multicolumn{8}{|c|}{ DD-ME $\delta$} \\
\hline 1.00 & 0.405 & 2.040 & 10.550 & 10.602 & 10.684 & 11.423 & 12.099 \\
\hline 1.44 & 0.552 & 2.890 & 10.913 & 10.945 & 10.996 & 11.442 & 11.837 \\
\hline 1.60 & 0.627 & 3.360 & 10.901 & 10.927 & 10.969 & 11.341 & 11.661 \\
\hline 1.96 & 1.214 & 7.938 & 9.843 & 9.855 & 9.876 & 10.052 & 10.194 \\
\hline \multicolumn{8}{|c|}{ DD-ME2 } \\
\hline 1.00 & 0.289 & 1.435 & 11.177 & 11.217 & 11.297 & 12.198 & 13.005 \\
\hline 1.44 & 0.347 & 1.755 & 12.021 & 12.049 & 12.104 & 12.716 & 13.234 \\
\hline 1.60 & 0.371 & 1.895 & 12.232 & 12.256 & 12.305 & 12.844 & 13.291 \\
\hline 2.49 & 0.817 & 5.345 & 11.717 & 11.725 & 11.743 & 11.931 & 12.073 \\
\hline \multicolumn{8}{|c|}{ IU-FSU } \\
\hline 1.00 & 0.333 & 1.665 & 10.931 & 11.014 & 11.082 & 11.777 & 12.616 \\
\hline 1.44 & 0.448 & 2.312 & 11.529 & 11.583 & 11.628 & 12.076 & 12.595 \\
\hline 1.60 & 0.508 & 2.676 & 11.586 & 11.634 & 11.672 & 12.053 & 12.482 \\
\hline 1.94 & 1.026 & 6.353 & 10.763 & 10.785 & 10.807 & 11.015 & 11.242 \\
\hline
\end{tabular}

the effect of $L$ on the density profile of the crust. Finally, in the last part we analyze the effect of finite temperature on the crust and the pasta phase. The discussion is done for neutron stars with masses $M=1 M_{\odot}, 1.44 M_{\odot}$, and $1.6 M_{\odot}$. The first two values have been chosen as representative masses since $1 M_{\odot}$ is smaller than the smallest neutron star mass detected until now and $1.44 M_{\odot}$ is the mass of the famous Hulse-Taylor pulsar. The value of $1.6 M_{\odot}$ is chosen to be slightly smaller than the maximum mass predicted by the FSU model. Results for the maximum neutron star mass configuration have also been obtained for all the models.

\section{A. Thickness and structure of inner crust}

We present here results for the pasta phase of $\beta$-equilibrium nonhomogeneous matter obtained within our TF calculation at $T=0$. As done by many authors, we assume that, for some given conditions (temperature, density, proton fraction, or chemical equilibrium), only a single geometry will be the physical one, i.e., the one with smaller free energy in comparison with homogeneous $\beta$-stable matter. At least five
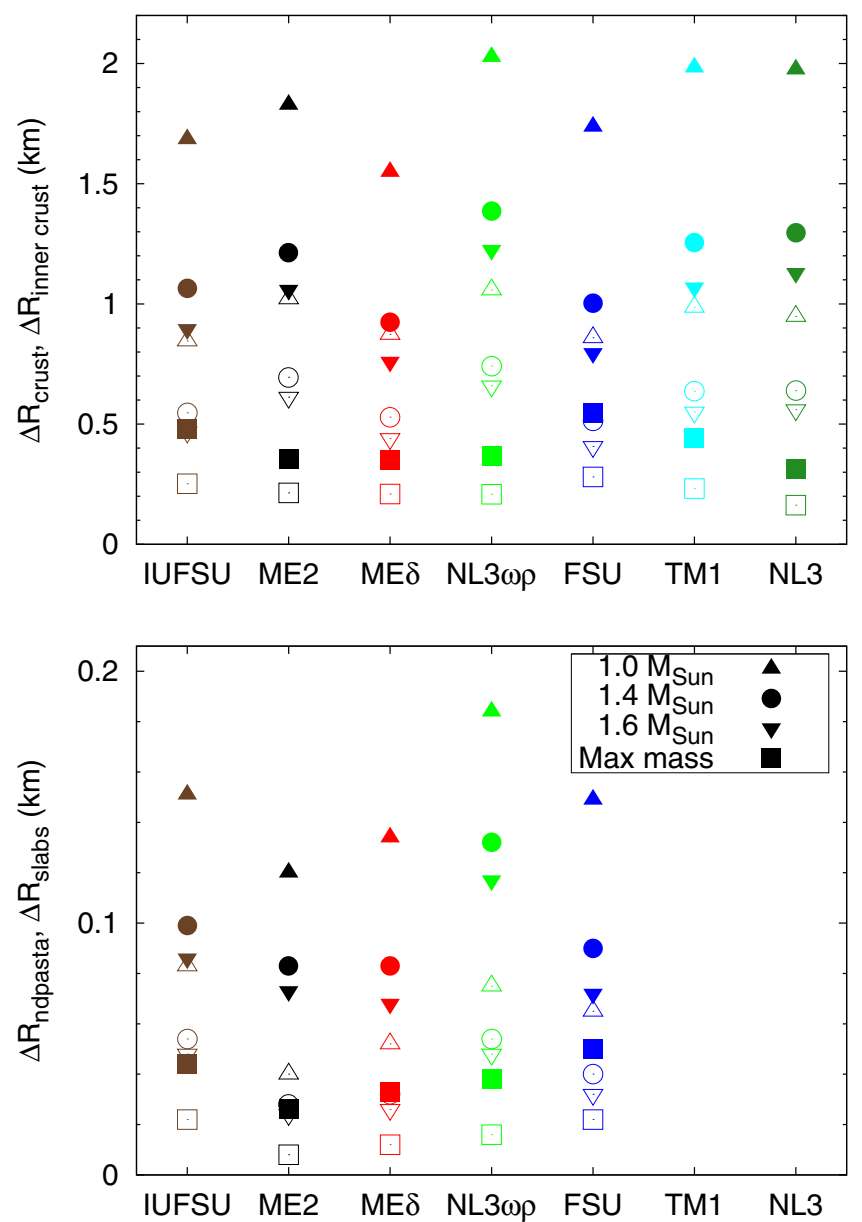

FIG. 1. (Color online) (top panel) Crust (full symbols) and innercrust (empty symbols) thickness. (bottom panel) Thickness of the nondroplet pasta (ndpasta; full symbols) and the slab (empty symbols) phases. The symbol shape identifies the star mass: $1 M_{\odot}$ (upward triangles), $1.44 M_{\odot}$ (circles), $1.6 M_{\odot}$ (downward triangles), and maximum mass (squares). 

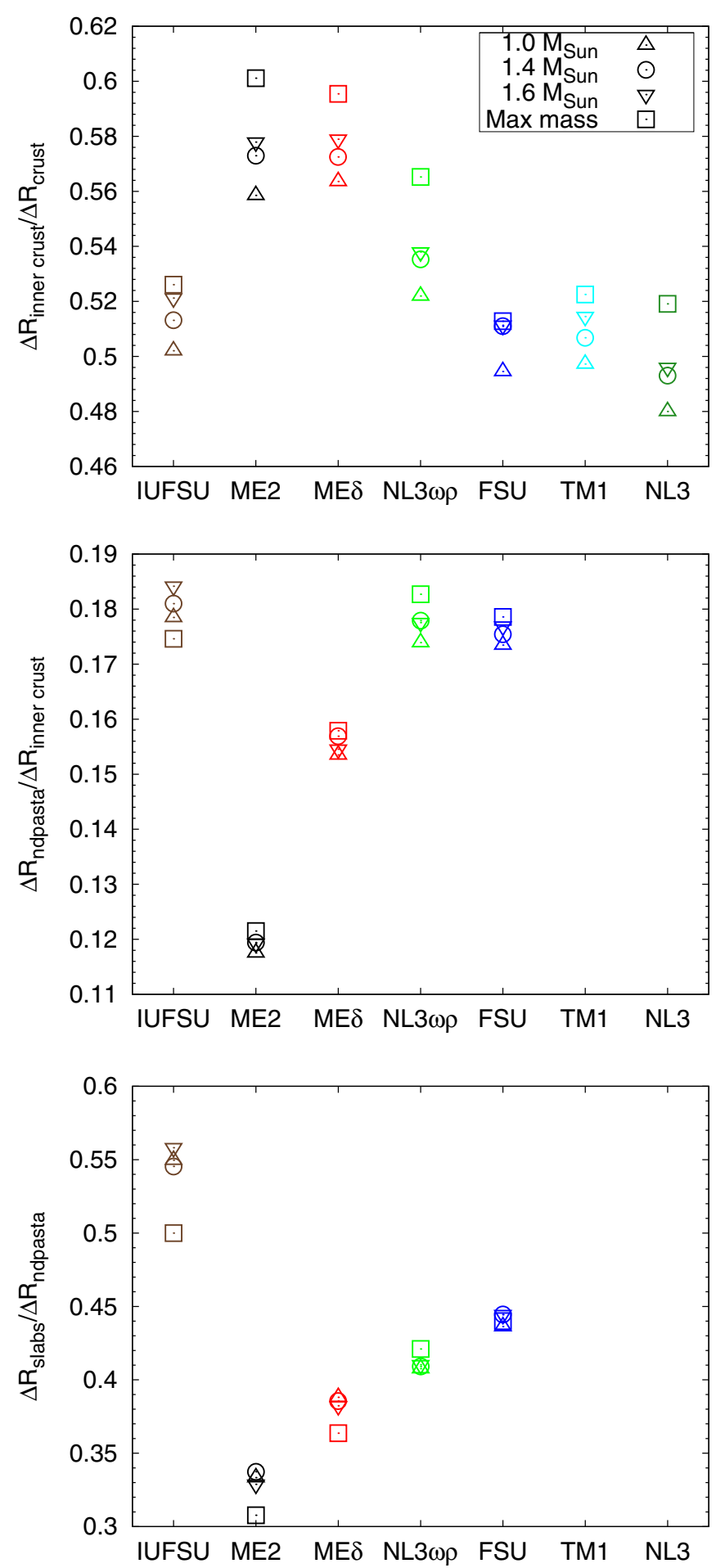

FIG. 2. (Color online) (top panel) Fraction of the crust occupied by the the inner crust. (middle panel) Fraction of the inner crust occupied by the nondroplet pasta (ndpasta) phase. (bottom panel) Fraction of the nondroplet pasta phase occupied by the slab phase. The symbol shapes have the same meaning as in Fig. 1.

different geometries could in principle occur: droplets, rods, slabs, tubes, and bubbles. However, due to the $\beta$ equilibrium the proton fraction is very small and only three of them are found to be energetically favorable: droplets, rods, and slabs.

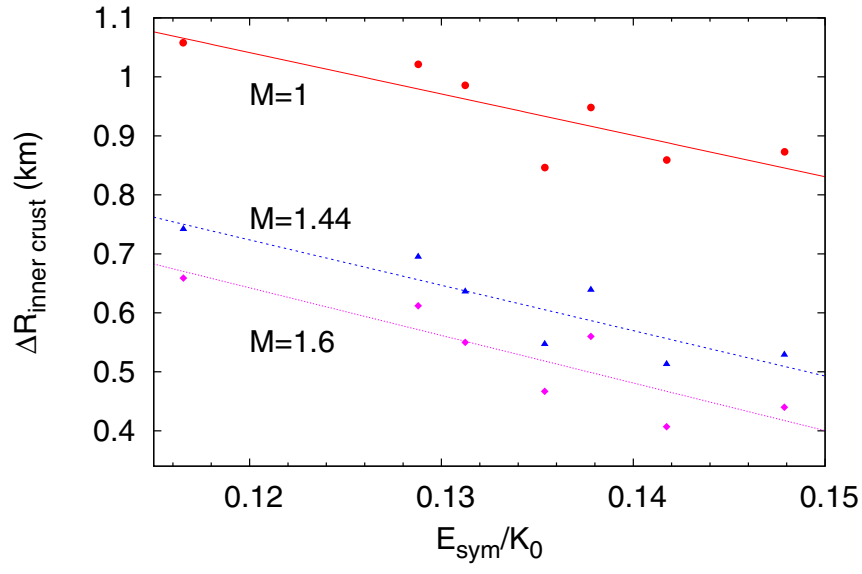

FIG. 3. (Color online) Correlation between the inner crust thickness and the ratio $E_{\text {sym }} / K_{0}$ for star with $M=1 M_{\odot}, 1.44 M_{\odot}, 1.6 M_{\odot}$. The slope of the three straight lines is similar $\sim-7.6 \pm 1.95 \mathrm{~km}$.

These structures form a regular lattice that we study in the WS approximation. The TF approach is semiclassical and does not include shell effects. Nevertheless, it was recently shown [12] that the main properties of the WS cells obtained within a TF calculation agree with Hartree-Fock (HF) [42] and Hartree-Fock-Boguliobov (HFB) [43] calculations, which allow the inclusion of shell effects. For a comparison of HFB and TF results the interested reader is referred to Ref. [12].

The complete stellar matter EoS is built by properly joining the inner-crust part with the outer-crust and the core parts. In this work, we assume that the core of the star is made only of nucleons, electrons, and muons, and its EoS is obtained also in the RMF approach imposing both $\beta$ equilibrium and charge neutrality. For the outer crust we consider the wellknown Baym-Pethick-Sutherland (BPS) EoS [44]. The TOV equations are then solved to determine the density profile of the neutron stars with the masses $M=1 M_{\odot}, 1.44 M_{\odot}, 1.6 M_{\odot}$, and $M_{\max }$ mentioned before.

In Table II we show for the different models some of the features of the inner-crust structure. All the models have a slab and a rod phase which together define the thickness of the nondroplet pasta, except for the NL3 and TM1 models. For these two models the inner crust is only formed by droplets in a neutron gas background. This is, as shown in Ref. [11], due to the high value of $L$ for these two models, 118 and $110 \mathrm{MeV}$, respectively.

In order to help the discussion, the results of Table II are also plotted in Figs. 1 and 2. In Fig. 1, the thickness of the total crust (full symbols) and inner crust (empty symbols) are shown in the top panel, and the thickness of the total nondroplet pasta phase (full symbols) and the slab phase (empty symbols) are plotted in the bottom panel. In Fig. 2 we plot the ratios of these quantities: the fraction of the inner crust with respect to the total crust (top panel), the fraction of the nondroplet pasta extension with respect to the total inner crust (middle panel) and the fraction of slab phase size with respect to the total nondroplet phase (bottom panel). The different models are ordered according to the magnitude of the slope $L$, which increases from left to right. 
TABLE III. Density transitions in the pasta phase at $T=0$ and melting temperature of the different pasta phases. For the transition to uniform matter we also show the values obtained with a dynamical spinodal calculation (DS).

\begin{tabular}{|c|c|c|c|c|c|c|c|}
\hline \multirow[t]{2}{*}{ Model } & \multicolumn{2}{|c|}{$\begin{array}{c}\rho_{\mathrm{h}-\mathrm{s}} \\
\left(\mathrm{fm}^{-3}\right)\end{array}$} & \multirow[t]{2}{*}{$\begin{array}{c}\rho_{\mathrm{s}-\mathrm{r}} \\
\left(\mathrm{fm}^{-3}\right)\end{array}$} & \multirow[t]{2}{*}{$\begin{array}{c}\rho_{\mathrm{r}-\mathrm{d}} \\
\left(\mathrm{fm}^{-3}\right)\end{array}$} & \multirow[t]{2}{*}{$\begin{array}{c}T_{s} \\
(\mathrm{MeV})\end{array}$} & \multirow[t]{2}{*}{$\begin{array}{c}T_{r} \\
(\mathrm{MeV})\end{array}$} & \multirow[t]{2}{*}{$\begin{array}{c}T_{m} \\
(\mathrm{MeV})\end{array}$} \\
\hline & $\mathrm{TF}$ & DS & & & & & \\
\hline NL3 & 0.0579 & 0.0550 & & & & & 4.7 \\
\hline TM1 & 0.0626 & 0.0602 & & & & & 5.1 \\
\hline FSU & 0.0751 & 0.0745 & 0.0673 & 0.0580 & 2.5 & 3.5 & 6.5 \\
\hline $\mathrm{NL}_{\omega \rho}$ & 0.0835 & 0.0838 & 0.0751 & 0.0642 & 3.0 & 3.5 & 5.8 \\
\hline DD-ME $\delta$ & 0.0766 & & 0.0720 & 0.0626 & 2.0 & 3.0 & 6.6 \\
\hline DD-ME2 & 0.0735 & & 0.0688 & 0.0611 & 1.0 & 2.0 & 5.4 \\
\hline IU-FSU & 0.0894 & 0.0855 & 0.0626 & 0.0471 & 9.0 & 9.5 & 9.8 \\
\hline
\end{tabular}

From Fig. 1 we see that no clear trend is found in the thickness of the different parts as a function of $L$. Instead, we found (see Fig. 3) that the size of the total crust is mainly defined by the incompressibility of the EoS (cf. Table I). In particular, the models NL3, NL3 $\omega \rho$, TM1, followed by DDME2, have the largest crusts and the largest incompressibilities. This is at variance with the results of Ref. [45] where, within the same model, the crust is calculated changing the isovector properties of the EoS; in particular, $E_{\text {sym }}$ and $L$. In this calculation the largest crusts occur for $L=70$ to $80 \mathrm{MeV}$. In general, a systematic behavior is observed with the mass of the star: the larger the mass, the thinner the crust and its sublayers. For instance, in all cases for $M=1 M_{\odot}$ the crust thickness lies between 1.5 and 2 $\mathrm{km}$, while for the maximum mass configurations it is mainly below $0.5 \mathrm{~km}$.

The top panel of Fig. 2 shows that the inner crust occupies $\sim 50 \%$ to $60 \%$ of the total crust size. The lower values were obtained with IUFSU, FSU, TM1, and NL3 with the smallest inner crust, $\sim 50 \%$, while the largest values occur with the density-dependent parametrizations. Once more, a systematic dependence upon the star mass is found: the fraction of the crust occupied by the inner crust increases with the star mass and there is a difference of $\sim+2 \%$ between a star with $1 M_{\odot}$ and $1.6 M_{\odot}$.
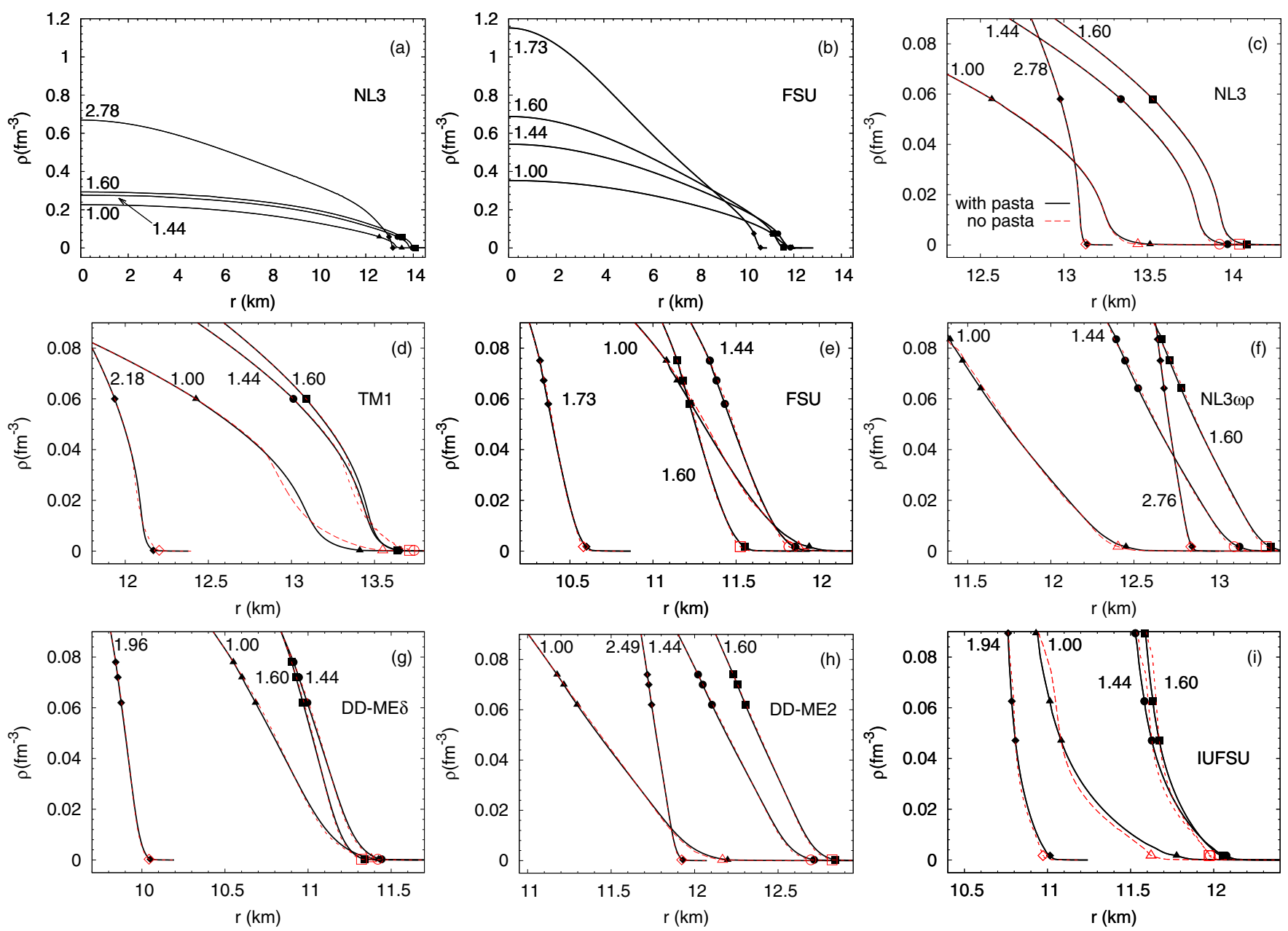

FIG. 4. (Color online) Profiles of neutron stars with a mass equal to $1 M_{\odot}, 1.44 M_{\odot}, 1.6 M_{\odot}$ and the maximum mass, for different nuclear models. The symbols stay at the the BPS-droplet, droplet-rod, rod-slab, slab-homogeneous transition for $1 M_{\odot}$ (triangles), 1.44 $M_{\odot}$ (circles), $1.6 M_{\odot}$ (squares), and maximum mass (diamonds). Panels (a) and (b) show the complete profile for NL3 and FSU, respectively. All other panels [(c)-(i)] focus on the crust profile. 
TABLE IV. Equation of state of the inner crust for the seven models considered. The energy density is given in units of $10^{-1} \mathrm{fm}^{-4}$, while the pressure is in units of $10^{-3} \mathrm{fm}^{-4}$.

\begin{tabular}{|c|c|c|c|c|c|c|c|}
\hline \multirow[t]{2}{*}{$\rho\left(\mathrm{fm}^{-3}\right)$} & NL3 & TM1 & FSU & $\mathrm{NL}_{\omega \rho}$ & DD-ME $\delta$ & DD-ME2 & IU-FSU \\
\hline & $\mathcal{E}$ & $\mathcal{E}$ & $\mathcal{E}$ & $\mathcal{E}$ & $\mathcal{E}$ & $\mathcal{E}$ & $\mathcal{E}$ \\
\hline
\end{tabular}

0.0860

0.0840

0.0820

0.0800

0.0780

0.0760

0.0740

0.0720

0.0700

0.0680

0.0660

0.0640

0.0620

0.0600

0.0580

0.0560

0.0540

0.0520

0.0500

0.0480

0.0460

0.0440

0.0420

0.0400

0.0380

0.0360

0.0340

0.0320

0.0300

0.0280

0.0260

0.0240

0.0220

0.0200

0.0180

0.0160

0.0140

0.0120

0.0100

0.0095

0.0090

0.0085

0.0080

0.0075

0.0070

0.0065

0.0060

0.0055

0.0050

0.0045

0.0040

0.0035

0.0030

0.0025

0.0020

$\begin{array}{lllll}3.94154 & 2.52403 & & \\ 3.84480 & 2.41543 & & \\ 3.74809 & 2.30744 & & \\ 3.65141 & 2.20122 & 3.65126 & 2.04088 \\ 3.55475 & 2.11172 & 3.55465 & 1.93136\end{array}$

4.139481 .60166 $4.04284 \quad 1.57348$ $3.94621 \quad 1.54348$ 3.849591 .50902 $3.75297 \quad 1.47400$ $3.65637 \quad 1.43842$ $3.55977 \quad 1.40239$

$\begin{array}{llllllllll}3.45832 & 1.86234 & 3.45812 & 2.01229 & 3.45807 & 1.82398 & 3.45856 & 2.06135 & 3.46319 & 1.36591\end{array}$ $\begin{array}{lllllllllll}3.36175 & 1.76038 & 3.36151 & 1.91484 & 3.36152 & 1.73271 & 3.36193 & 1.95675 & 3.36661 & 1.32916\end{array}$ $\begin{array}{llllllllll}3.26521 & 1.66110 & 3.26494 & 1.81906 & 3.26500 & 1.63419 & 3.26533 & 1.86477 & 3.27005 & 1.29192\end{array}$ $\begin{array}{lllllllllll}3.16870 & 1.57890 & 3.16839 & 1.72541 & 3.16850 & 1.53902 & 3.16875 & 1.76524 & 3.17350 & 1.25411\end{array}$ $\begin{array}{llllllllll}3.07221 & 1.48951 & 3.07187 & 1.63348 & 3.07203 & 1.44694 & 3.07221 & 1.66744 & 3.07696 & 1.21646\end{array}$ $\begin{array}{llllllllll}2.97575 & 1.40336 & 2.97537 & 1.54804 & 2.97559 & 1.35825 & 2.97570 & 1.57160 & 2.98043 & 1.17734\end{array}$ $\begin{array}{llllllllll}2.87932 & 1.31999 & 2.87891 & 1.46017 & 2.87918 & 1.27656 & 2.87922 & 1.48170 & 2.88391 & 1.15722\end{array}$ $\begin{array}{llllllllllll}2.77736 & 1.48251 & 2.78292 & 1.24043 & 2.78247 & 1.37452 & 2.78280 & 1.19477 & 2.78276 & 1.39043 & 2.78740 & 1.11951\end{array}$ $\begin{array}{llllllllllllll}2.67959 & 1.36758 & 2.68110 & 1.33925 & 2.68654 & 1.16654 & 2.68607 & 1.29055 & 2.68644 & 1.11632 & 2.68634 & 1.30149 & 2.69090 & 1.08100\end{array}$ $\begin{array}{llllllllllllll}2.58343 & 1.24190 & 2.58489 & 1.20460 & 2.59019 & 1.09281 & 2.58969 & 1.20876 & 2.59011 & 1.04117 & 2.58995 & 1.21468 & 2.59442 & 1.04177\end{array}$

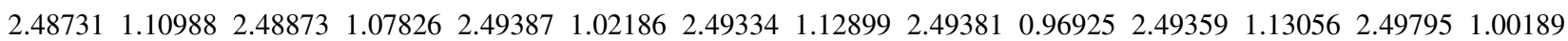

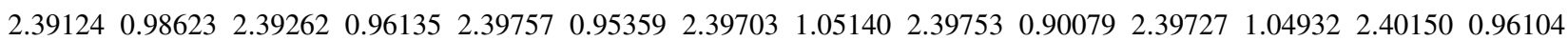
$\begin{array}{llllllllllllll}2.29522 & 0.87140 & 2.29656 & 0.85336 & 2.30130 & 0.88792 & 2.30074 & 0.97589 & 2.30129 & 0.83526 & 2.30097 & 0.97047 & 2.30506 & 0.91959\end{array}$ $\begin{array}{llllllllllllll}2.19925 & 0.76548 & 2.20053 & 0.75398 & 2.20505 & 0.82493 & 2.20448 & 0.90271 & 2.20506 & 0.77288 & 2.20471 & 0.89456 & 2.20864 & 0.88260\end{array}$

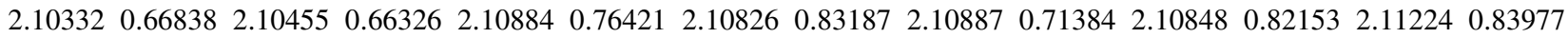

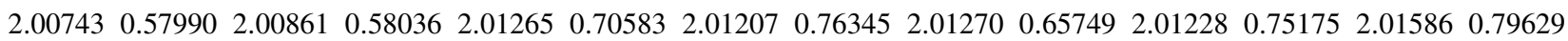
$\begin{array}{llllllllllllll}1.91158 & 0.49988 & 1.91270 & 0.50581 & 1.91648 & 0.65009 & 1.91591 & 0.69757 & 1.91656 & 0.60433 & 1.91612 & 0.68485 & 1.91950 & 0.75215\end{array}$

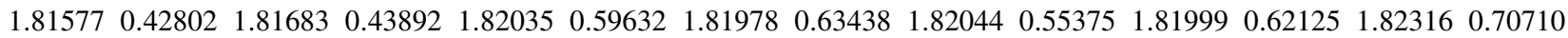
$\begin{array}{llllllllllllll}1.71999 & 0.36366 & 1.72099 & 0.37922 & 1.72424 & 0.54503 & 1.72368 & 0.57417 & 1.72435 & 0.50611 & 1.72389 & 0.56110 & 1.72684 & 0.66164\end{array}$ $\begin{array}{llllllllllllll}1.62425 & 0.30710 & 1.62519 & 0.32641 & 1.62816 & 0.49573 & 1.62762 & 0.51660 & 1.62828 & 0.46111 & 1.62782 & 0.50414 & 1.63055 & 0.61573\end{array}$ $\begin{array}{llllllllllllll}1.52854 & 0.25764 & 1.52941 & 0.27974 & 1.53211 & 0.44880 & 1.53159 & 0.46202 & 1.53224 & 0.41839 & 1.53179 & 0.45077 & 1.53429 & 0.56936\end{array}$

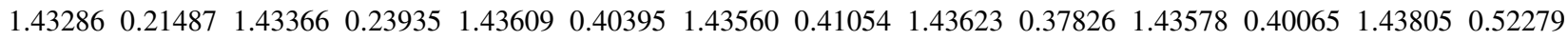
$\begin{array}{lllllllllllllll}1.33721 & 0.17828 & 1.33793 & 0.20443 & 1.34009 & 0.36143 & 1.33963 & 0.36219 & 1.34024 & 0.34020 & 1.33981 & 0.35413 & 1.34185 & 0.47616\end{array}$ $\begin{array}{llllllllllllll}1.24158 & 0.14742 & 1.24223 & 0.17453 & 1.24413 & 0.32094 & 1.24370 & 0.31724 & 1.24428 & 0.30452 & 1.24388 & 0.31080 & 1.24568 & 0.42990\end{array}$ $\begin{array}{llllllllllllll}1.14597 & 0.12168 & 1.14655 & 0.14904 & 1.14819 & 0.28293 & 1.14781 & 0.27538 & 1.14835 & 0.27072 & 1.14797 & 0.27102 & 1.14955 & 0.38403\end{array}$ $\begin{array}{llllllllllllll}1.05038 & 0.10070 & 1.05089 & 0.12750 & 1.05229 & 0.24695 & 1.05194 & 0.23687 & 1.05244 & 0.23894 & 1.05210 & 0.23433 & 1.05345 & 0.33888\end{array}$ $\begin{array}{llllllllllllll}0.95481 & 0.08382 & 0.95525 & 0.10921 & 0.95642 & 0.21340 & 0.95611 & 0.20175 & 0.95656 & 0.20879 & 0.95625 & 0.20094 & 0.95740 & 0.29484\end{array}$ $\begin{array}{llllllllllllll}0.85925 & 0.07034 & 0.85962 & 0.09385 & 0.86058 & 0.18198 & 0.86032 & 0.16967 & 0.86071 & 0.18046 & 0.86044 & 0.17033 & 0.86138 & 0.25222\end{array}$ $\begin{array}{lllllllllllllll}0.76371 & 0.05970 & 0.76401 & 0.08068 & 0.76478 & 0.15294 & 0.76455 & 0.14068 & 0.76489 & 0.15360 & 0.76467 & 0.14261 & 0.76542 & 0.21158\end{array}$ $\begin{array}{llllllllllllll}0.66817 & 0.05118 & 0.66842 & 0.06917 & 0.66901 & 0.12598 & 0.66883 & 0.11489 & 0.66910 & 0.12837 & 0.66892 & 0.11747 & 0.66950 & 0.17301\end{array}$ $\begin{array}{llllllllllllll}0.57265 & 0.04399 & 0.57284 & 0.05873 & 0.57327 & 0.10120 & 0.57313 & 0.09162 & 0.57335 & 0.10445 & 0.57321 & 0.09461 & 0.57364 & 0.13703\end{array}$ $\begin{array}{lllllllllllllll}0.47714 & 0.03760 & 0.47697 & 0.04799 & 0.47758 & 0.07870 & 0.47748 & 0.07095 & 0.47764 & 0.08210 & 0.47754 & 0.07389 & 0.47783 & 0.10429\end{array}$ $\begin{array}{llllllllllllll}0.45327 & 0.03608 & 0.45310 & 0.04561 & 0.45366 & 0.07338 & 0.45357 & 0.06618 & 0.45372 & 0.07673 & 0.45362 & 0.06897 & 0.45389 & 0.09659\end{array}$ $\begin{array}{llllllllllllll}0.42939 & 0.03451 & 0.42923 & 0.04318 & 0.42975 & 0.06816 & 0.42966 & 0.06157 & 0.42980 & 0.07145 & 0.42971 & 0.06421 & 0.42995 & 0.08909\end{array}$ $\begin{array}{llllllllllllll}0.40552 & 0.03289 & 0.40536 & 0.04074 & 0.40584 & 0.06309 & 0.40576 & 0.05706 & 0.40588 & 0.06624 & 0.40581 & 0.05955 & 0.40602 & 0.08184\end{array}$ $\begin{array}{lllllllllllllll}0.38165 & 0.03132 & 0.38149 & 0.03836 & 0.38193 & 0.05818 & 0.38186 & 0.05270 & 0.38197 & 0.06122 & 0.38190 & 0.05504 & 0.38209 & 0.07485\end{array}$ $\begin{array}{llllllllllllll}0.35777 & 0.02970 & 0.35763 & 0.03588 & 0.35802 & 0.05336 & 0.35796 & 0.04840 & 0.35806 & 0.05620 & 0.35800 & 0.05058 & 0.35816 & 0.06806\end{array}$ $\begin{array}{llllllllllllll}0.33390 & 0.02797 & 0.33376 & 0.03340 & 0.33412 & 0.04870 & 0.33407 & 0.04429 & 0.33415 & 0.05139 & 0.33410 & 0.04632 & 0.33424 & 0.06152\end{array}$ $\begin{array}{lllllllllllllll}0.31003 & 0.02630 & 0.30990 & 0.03096 & 0.31022 & 0.04414 & 0.31017 & 0.04024 & 0.31025 & 0.04657 & 0.31021 & 0.04211 & 0.31033 & 0.05529\end{array}$ $\begin{array}{llllllllllllll}0.28617 & 0.02453 & 0.28604 & 0.02853 & 0.28633 & 0.03978 & 0.28629 & 0.03639 & 0.28635 & 0.04196 & 0.28631 & 0.03801 & 0.28642 & 0.04916\end{array}$ $\begin{array}{llllllllllllll}0.26230 & 0.02270 & 0.26218 & 0.02605 & 0.26243 & 0.03552 & 0.26240 & 0.03264 & 0.26246 & 0.03750 & 0.26242 & 0.03406 & 0.26251 & 0.04338\end{array}$ $\begin{array}{lllllllllllllll}0.23843 & 0.02088 & 0.23832 & 0.02351 & 0.23855 & 0.03137 & 0.23852 & 0.02899 & 0.23856 & 0.03309 & 0.23854 & 0.03015 & 0.23861 & 0.03791\end{array}$ $\begin{array}{llllllllllllll}0.21457 & 0.01895 & 0.21447 & 0.02108 & 0.21466 & 0.02742 & 0.21464 & 0.02544 & 0.21468 & 0.02889 & 0.21466 & 0.02645 & 0.21471 & 0.03269\end{array}$ $\begin{array}{lllllllllllllll}0.19071 & 0.01703 & 0.19061 & 0.01855 & 0.19078 & 0.02362 & 0.19076 & 0.02204 & 0.19079 & 0.02488 & 0.19078 & 0.02286 & 0.19082 & 0.02777\end{array}$ $\begin{array}{lllllllllllllll}0.16685 & 0.01500 & 0.16677 & 0.01606 & 0.16691 & 0.02002 & 0.16689 & 0.01880 & 0.16692 & 0.02098 & 0.16690 & 0.01941 & 0.16694 & 0.02316\end{array}$

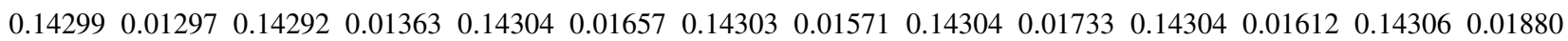
$\begin{array}{llllllllllllll}0.11914 & 0.01095 & 0.11908 & 0.01125 & 0.11917 & 0.01333 & 0.11916 & 0.01272 & 0.11918 & 0.01389 & 0.11917 & 0.01297 & 0.11919 & 0.01485\end{array}$ \begin{tabular}{lllllllllllllll}
0.09529 & 0.00892 & 0.09524 & 0.00892 & 0.09532 & 0.01034 & 0.09531 & 0.00998 & 0.09532 & 0.01069 & 0.09531 & 0.01014 & 0.09533 & 0.01125 \\
\hline
\end{tabular} 
TABLE IV. (Continued.)

\begin{tabular}{|c|c|c|c|c|c|c|c|c|c|c|c|c|c|}
\hline$\rho\left(\mathrm{fm}^{-3}\right)$ & \multicolumn{2}{|c|}{ NL3 } & \multicolumn{2}{|c|}{ TM1 } & \multicolumn{2}{|c|}{ FSU } & \multicolumn{2}{|c|}{$\mathrm{NL}_{\omega \rho}$} & \multicolumn{2}{|c|}{ DD-ME $\delta$} & \multicolumn{2}{|c|}{ DD-ME2 } & IU-FSU \\
\hline
\end{tabular}

$\begin{array}{lllllllllllllllll}0.0015 & 0.07145 & 0.00694 & 0.07141 & 0.00674 & 0.07146 & 0.00755 & 0.07146 & 0.00745 & 0.07147 & 0.00780 & 0.07146 & 0.00745 & 0.07147 & 0.00806\end{array}$ $\begin{array}{lllllllllllllll}0.0010 & 0.04761 & 0.00507 & 0.04759 & 0.00466 & 0.04762 & 0.00517 & 0.04762 & 0.00517 & 0.04762 & 0.00532 & 0.04762 & 0.00507 & 0.04763 & 0.00537\end{array}$ $\begin{array}{llllllllllllllll}0.0009 & 0.04285 & 0.00466 & 0.04283 & 0.00431 & 0.04286 & 0.00476 & 0.04285 & 0.00476 & 0.04285 & 0.00487 & 0.04286 & 0.00466 & 0.04286 & 0.00487\end{array}$ $\begin{array}{llllllllllllllll}0.0008 & 0.03808 & 0.00431 & 0.03806 & 0.00395 & 0.03809 & 0.00431 & 0.03809 & 0.00436 & 0.03809 & 0.00441 & 0.03809 & 0.00426 & 0.03809 & 0.00441\end{array}$ $\begin{array}{llllllllllllllll}0.0007 & 0.03332 & 0.00395 & 0.03330 & 0.00360 & 0.03332 & 0.00395 & 0.03332 & 0.00400 & 0.03332 & 0.00400 & 0.03332 & 0.00385 & 0.03332 & 0.00400\end{array}$ $\begin{array}{llllllllllllllll}0.0006 & 0.02855 & 0.00365 & 0.02854 & 0.00324 & 0.02856 & 0.00355 & 0.02855 & 0.00360 & 0.02856 & 0.00360 & 0.02856 & 0.00350 & 0.02856 & 0.00355\end{array}$ $\begin{array}{lllllllllllllll}0.0005 & 0.02379 & 0.00334 & 0.02378 & 0.00289 & 0.02379 & 0.00314 & 0.02379 & 0.00324 & 0.02379 & 0.00324 & 0.02379 & 0.00314 & 0.02379 & 0.00319\end{array}$ $\begin{array}{llllllllllllllll}0.0004 & 0.01902 & 0.00299 & 0.01902 & 0.00258 & 0.01903 & 0.00284 & 0.01903 & 0.00289 & 0.01903 & 0.00289 & 0.01903 & 0.00279 & 0.01903 & 0.00284\end{array}$ $\begin{array}{lllllllllllllll}0.0003 & 0.01426 & 0.00258 & 0.01426 & 0.00228 & 0.01426 & 0.00248 & 0.01426 & 0.00253 & 0.01426 & 0.00253 & 0.01426 & 0.00243 & 0.01426 & 0.00248\end{array}$

Next, we come to the size of the nondroplet pasta phase: the phases that correspond to a frustrated system. In a $1 M_{\odot}$ star the nondroplet pasta size is smaller than $200 \mathrm{~m}$ (Fig. 1, bottom). Its relative size is slightly smaller in less massive stars (Fig. 2, middle). Within the nondroplet pasta phase, we find in our results slab and rod phases. The slab fraction corresponds to $\sim 35 \%$ to $40 \%$ of the total nondroplet pasta phase for all the models, apart from IU-FSU, where it is almost 55\% and DDME2 with $\sim 30 \%$ to $45 \%$ (Fig. 2, bottom). The different behavior of IU-FSU is mainly due to the small proton fraction in the cluster. IU-FSU has a small value of the symmetry energy slope at subsaturation densities, which affects the surface tension giving quite a high surface tension; see Ref. [12], and preventing the neutron drip. A small proton fraction in the cluster favors the slab geometry with respect to the rod geometry because the surface energy decreases with the proton fraction. On the contrary, a smaller surface tension favors the neutron drip. Clusters are more isospin symmetric and the droplet geometry is favored. In Ref. [12] it is shown that the DDME2 and NL3 models have the smallest surface energies for nuclear symmetric matter. This could explain the behavior of DDME2 which has the smallest fraction of the nondroplet pasta phase.

In Ref. [45] the effect of the nuclear pasta on the crustal shear phenomena was studied. In particular, two limits were considered; namely, the pasta as an elastic solid and as a liquid. In the first case the shear modulus was calculated at the crust-core transition while in the second case it was done at the transition from the droplet phase to the nondroplet pasta phase. For models with no nondroplet pasta phase, such as NL3 and TM1, there is no difference between these two pictures. However, models with a symmetry energy slope $L$ below $80 \mathrm{MeV}$ have a nondroplet pasta phase, and the ratio of shear modulus to pressure can be as high as two times larger if the pasta is considered an elastic solid and $L=40 \mathrm{MeV}$. An intermediate picture would consider the rod phase as an elastic solid and the slab phase as a liquid phase.

Comparing our results with those discussed in Ref. [45], a couple of comments are in order. First, the incompressibility of the EoS seems to have an important influence on the total crust, so that DDME2 with $L=51$ and $K_{0}=250.8 \mathrm{MeV}$ has a larger crust than FSU with $L=60$ and $K_{0}=230 \mathrm{MeV}$. Second, except for NL3 $\omega \rho$ with a large $K_{0}$, all the other models predict the nondroplet pasta phase of the $1.44 M_{\odot}$ star with a thickness of $\sim 80 \mathrm{~m}$, similar to that calculated in Ref. [45].

Crustal thicknesses were determined in several recent papers $[18,19,39,45,46]$. In these works the crust-core boundary, essential to determine the crustal thickness, was obtained from the instability against small-amplitude density fluctuations [18,39] or the compressible liquid drop model (CLDM) $[19,45,46]$. All the works consider the BPS EoS for the outer crust. The inner crust EoS was obtained within the CLDM and the Wigner-Seitz approximation in Refs. [45,46] and a polytropic component of the EoS that interpolates between the neutron drip density and the crust-core density $[18,39]$.

Comparing our results for the crust thickness to those of the above works we conclude that there is an overall agreement. For $M=1.44 M_{\odot}$ our crust thickness lies between $0.892 \mathrm{~km}$ for DDME2 (the only model giving a thickness below $1 \mathrm{~km}$ ) and $1.386 \mathrm{~km}$ for NL3 $\omega \rho$ with $L=55 \mathrm{MeV}$. Our result for IUFSU agrees with Ref. [18].

Within the set of models chosen we do not get a thickness as large as those obtained in Ref. [18], comparing with the $1.4 M_{\odot}$ results. This could be due to the fact that the authors study the parametrizations that give the largest pressures at the crust core, which are close to some of the parametrizations of the present study but not the same. However, in Ref. [39] some of the same parametrizations we consider are studied by using the same method and larger thicknesses than ours are obtained: $2.03,1.76$, and $1.48 \mathrm{~km}$ for NL3 $\omega \rho$ with $\Lambda_{v}=$ 0.03 , NL3, and FSU, respectively. These numbers should be compared with the numbers we obtain: $1.39,1.30$, and $1.00 \mathrm{~km}$, respectively. The method applied by these authors to determine the crust-core transition gives results very similar to the Thomas-Fermi approximation; see Table III. Therefore, it could be that the polytropic parametrization of the innercrust EoS is the responsible for the differences in the crust thicknesses.

Next we compare our calculations of crust thickness with results obtained from a systematic study that uses neutron star observations [19]. We must restrict ourselves to the models with $L<60 \mathrm{MeV}$, i.e., we exclude TM1 and NL3. For all other models the crust-core transition density $\rho_{t}$ lies within $0.0751 \mathrm{fm}^{-3}$ (FSU) and $0.0855 \mathrm{fm}^{-3}$ (IUFSU) 
and, therefore, we take the numbers given in Ref. [19] for $\rho_{t}=0.08 \mathrm{fm}^{-3}$. Our values are compatible with those obtained by using a quantum Monte Carlo EoS calculated with chiral effective theory interactions, at saturation density or below and above saturation density an EoS formed by three piecewise polytropes, designed by model $\mathrm{A}$ in Ref. [19].

As mentioned before, both the incompressibility and the density dependence of the symmetry energy affect the size of the inner crust. We obtained a possible correlation between the ratio $E_{\text {sym }} / K_{0}$ and the inner-crust thickness. This correlation is shown in Fig. 3. This should be further investigated and confirmed with a larger set of models. The correlation is worse for the $1.6 M_{\odot}$ star, probably because this mass lies closer to the maximum-mass configuration. The slope obtained for the three star masses analyzed is $\sim-7.6 \pm 1.95 \mathrm{~km}$.

\section{B. Density profile of crust}

In Fig. 4 we present the profile of stars with masses $M=$ $1 M_{\odot}, 1.44 M_{\odot}, 1,60 M_{\odot}$, and $M_{\max }$. The whole star profile is shown for the models NL3 and FSU in Figs. 4(a) and 4(b), respectively, since these two models predict the largest and the smallest maximum-mass configuration.

All the other panels of Fig. 4 show, instead, for the whole set of models under discussion, only the last $\sim 2 \mathrm{~km}$ of the star profile close to the surface. The results determined with the same EoS presented in the previous section (i.e., including the TF calculation of the inner crust) are represented by a solid black line, and the transitions between the different phases of the inner crust are identified with full black symbols. For comparison, the result obtained joining the BPS EoS directly to the homogeneous stellar matter EoS (red dashed lines) is also shown. In this case, the transition from the BPS to the homogeneous matter is shown by a red empty symbol. The EoSs of the inner crust obtained within the TF framework and used to calculate the crust profiles are given in Table IV.

Within the same model, a larger mass corresponds to a steeper profile, as expected, due to the larger gravitational force. In models with a large incompressibility, like NL3, TM1, NL3 $\omega \rho$, and DDME2, and taking only the $1 M_{\odot}, 1.44 M_{\odot}$, and $1.6 M_{\odot}$ stars, the star with the largest mass has the inner crust at a larger distance from the center. On the contrary, in the case of FSU, IU-FSU, and DDME $\delta$ there is a larger concentration of mass at the center because the EoS is softer, and the crust is pushed more strongly towards the center of the star: this explains why, for IU-FSU and DDME $\delta$, the profiles of the $1.44 M_{\odot}$ and $1.6 M_{\odot}$ stars are almost coincident, and for FSU the profiles of the $1 M_{\odot}$ and $1.44 M_{\odot}$ stars cross, while the crust of the $1.6 M_{\odot}$ one has the smallest distance to the star center. Notice that, for NL3, TM1, and DDME2, the maximum-mass star also has the smallest radius.

One interesting conclusion is that taking into account the correct description of the inner crust in the total stellar EoS is more important for the softer EoS and with smaller slopes $L$. However, on the whole, using the BPS EoS for the outer crust and an EoS of homogeneous stellar matter for the inner crust and core gives good results for the stellar profiles.
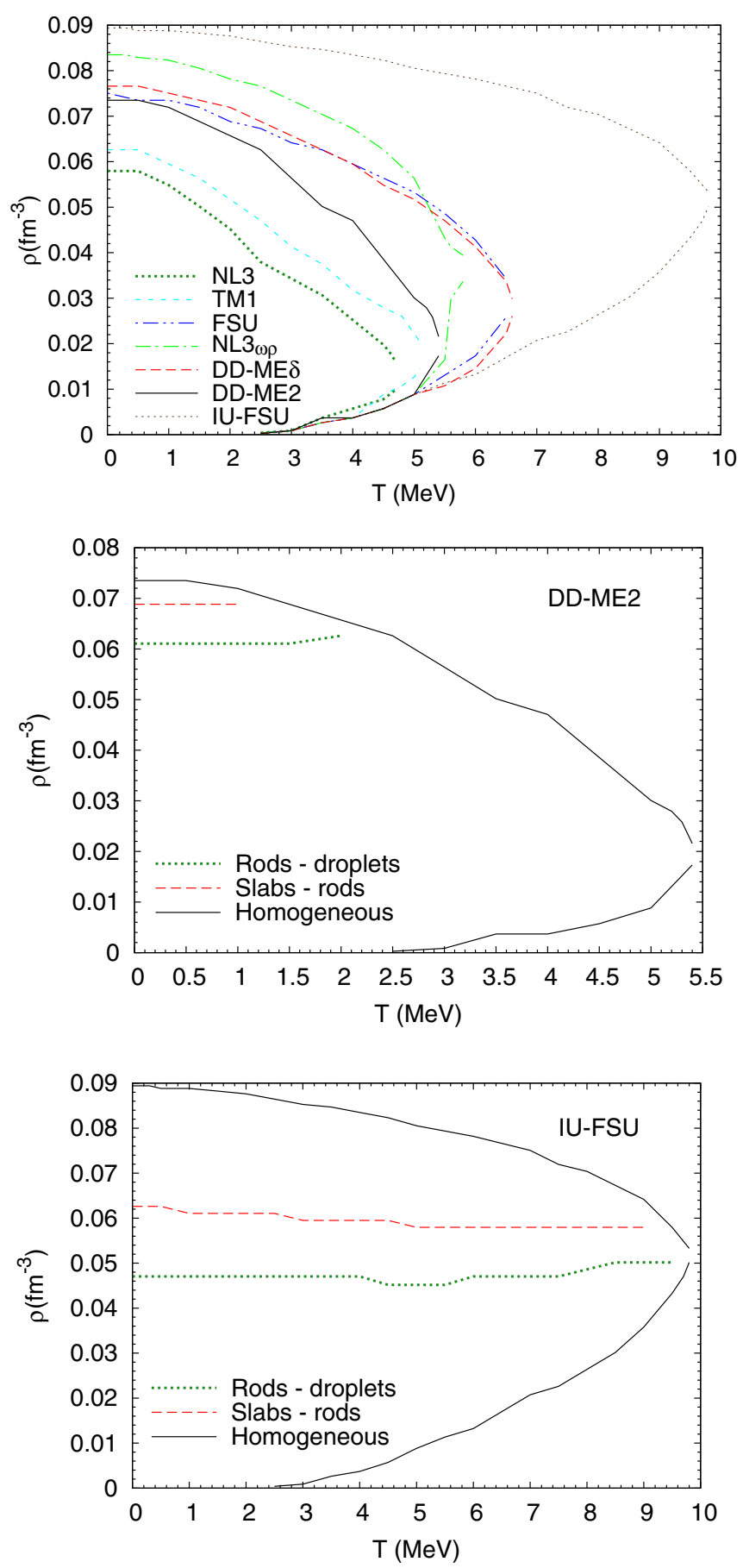

FIG. 5. (Color online) (top panel) Density range of the crust as a function of temperature for all models considered in the present study. (middle and bottom panels) Size of the pasta phases versus $T$ for DDME2 and IU-FSU. In these two panels, the dotted (dashed) line represents the transition droplet rod (rod slab).

\section{Finite-temperature effects on crust}

In this section we present the results of a finite-temperature calculation of the crust size for $\beta$-equilibrium matter. Our main objectives are (i) to determine the critical temperatures below which clusterization should be taken into account; (ii) to verify how temperature affects the pasta phase, 


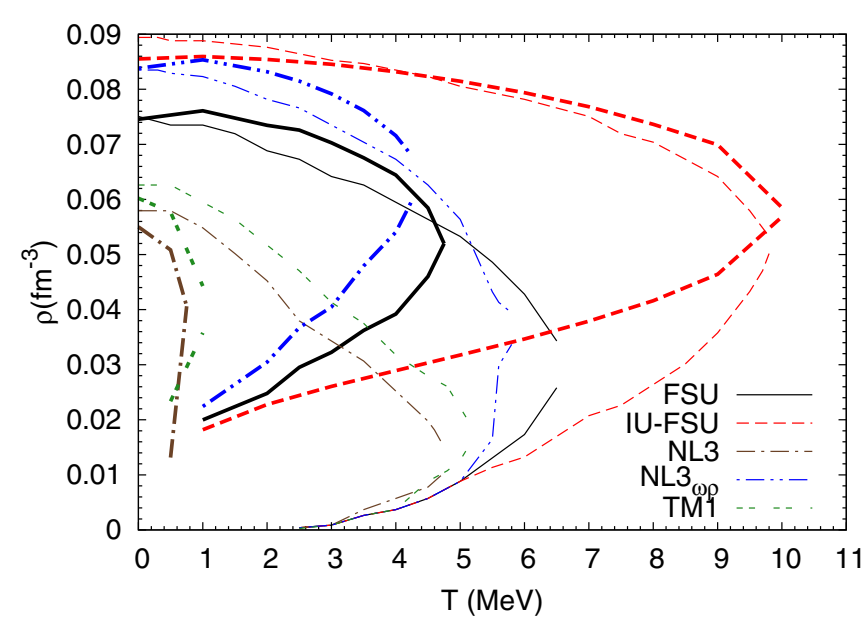

FIG. 6. (Color online) (top panel) Density range of the crust as a function of temperature for some of the relativistic mean-field models considered in the present study, obtained by using a TF calculation (thin lines) and a DS calculation (thick lines).

particularly the transition between the different geometries and the size of the cells; and (iii) to determine the melting temperature of clusters with different geometry.

We perform the study by using both a finite-temperature TF calculation of the pasta phase and the finite-temperature dynamical approach within the relativistic Vlasov formalism [24-26,47]. In the latter case, the crust-core transition is defined by the intersection between the $\beta$-equilibrium EoS and the dynamical spinodal (DS). The cluster size is identified with half the wavelength of the density fluctuation $[24,25,27]$. The results are shown in Fig. 5 for the TF calculation; a comparison between the TF and the DS calculation is done in Fig. 6 and in Table III.

The critical temperature is model dependent and both the density dependence of the symmetry energy and the incompressibility affect this quantity: a smaller value of $L$ and a smaller $K_{0}$ favor clusterization at larger temperatures. The results obtained within the TF approach are compatible with the estimations calculated by using the dynamical calculation at the bottom of the inner crust [9]. Close to the outer crust the dynamical calculations estimations give much larger densities. This could be expected, considering that in the TF calculation the inner crust in this region is formed by small droplets inside cells with a much larger radius. On the other hand, the dynamical calculation considers always that the cluster size is half the WS cell and, therefore, has a much larger surface-energy contribution. Our results are compatible with those obtained in Ref. [27] (see Fig. 11 of this reference), where most of the results have been obtained for Skyrme forces.

From the middle and lower panels of Fig. 5, we observe that the droplet-rod and the rod-slab transition densities do not depend on the temperature. However, the melting temperature of the three geometries is different, with the droplets surviving up to higher temperatures.

In the present calculation we suppose that the WS cells exist until the clusters melt. This is an approximation that will probably break down close to the melting point due to thermal fluctuations and, therefore, the numbers obtained should be interpreted as an upper limit. The problem of the effect of thermal fluctuations on the pasta structures was studied in Refs. [28,29] and it was shown that thermally induced displacements of the rod-like and slab-like nuclei can melt the lattice structure when these displacements are larger than the space available between the cluster and the
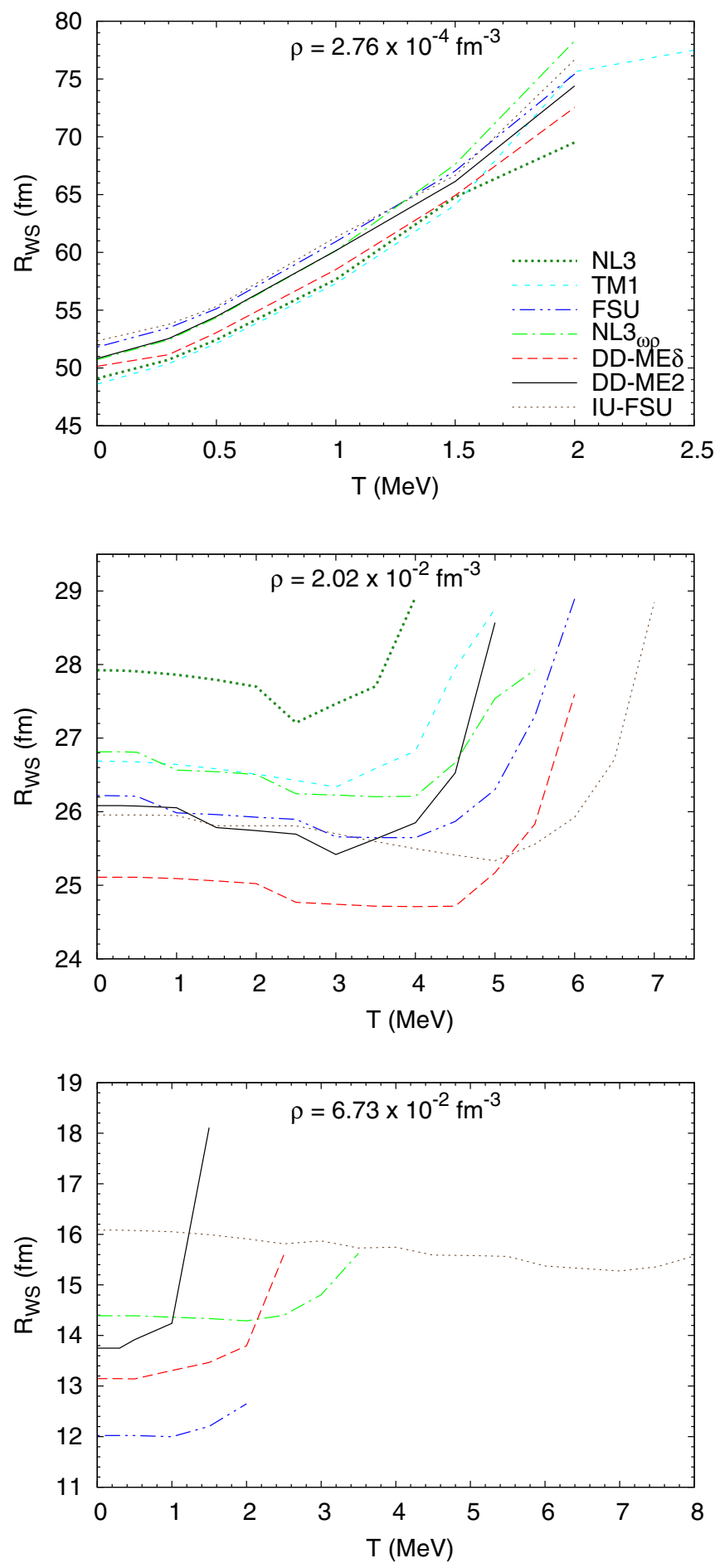

FIG. 7. (Color online) Radius of the cells as a function of the temperature at the densities reported at the top of the panels. 
boundary of the WS cell. Moreover, it was also shown that slab-like nuclei would more easily be dissolved, while the rod like were expected to survive at temperatures relevant for supernova cores. In the present calculation, except for IU-FSU, all models predict the melting of the slabs at a temperature $T<3 \mathrm{MeV}$, while the rods will melt at temperatures $1 \mathrm{MeV}$ higher; see Table III. Neglecting thermal fluctuations, we expect that clusters will survive in $\beta$-equilibrium matter at temperatures below 5 to $6 \mathrm{MeV}$, approximately twice the melting temperature of nondroplet structures. According to the results of Ref. [21] the appearance of the nonspherical pasta structures will affect the cooling by making matter more transparent to low-energy neutrino diffusion and increase the structure factor for larger-energy neutrinos.

We next analyze the effect of the temperature on the size of the WS cells. In Fig. 7 we plot the WS radius as a function of the temperature for all the models. We select three reference densities: $\rho=2.76 \times 10^{-4}$ and $2.02 \times 10^{-2}$ $\mathrm{fm}^{-3}$, at which the clusters are spherical for all models, and $\rho=6.73 \times 10^{-2} \mathrm{fm}^{-3}$, where all are in rod phase except IU-FSU, which is in a slab phase. In Fig. 8 we focus on the TM1 model, which only shows spherical clusters, and plot the radius of the WS cell versus density at different temperatures.

In general, for the densities shown, the WS radius increases with the temperature, but for $\rho=2.02 \times 10^{-2} \mathrm{fm}^{-3}$ the $R_{\mathrm{WS}}$ suffers a small decrease of the order of $0.5 \mathrm{fm}$ below $T=3$ to 4 $\mathrm{MeV}$. The surface energy decreases with temperature and, as a result, we could expect that the Wigner-Seitz radius would decrease with temperature. This, in fact, occurs for temperatures well below the critical transition temperature to homogeneous matter, corresponding to the behavior below $T \lesssim 4 \mathrm{MeV}$ in Fig. 7, middle panel for most of the models considered, or to the IUFSU below $7 \mathrm{MeV}$ and NL3 $\omega \rho$ below $2 \mathrm{MeV}$ in the bottom panel of Fig. 7. However, for temperatures close to the critical temperature, the Wigner-Seitz radius increases most probably due to the restrictions of the present calculation which does not allow the freedom for the cluster to choose the shape that minimizes the free energy. A similar

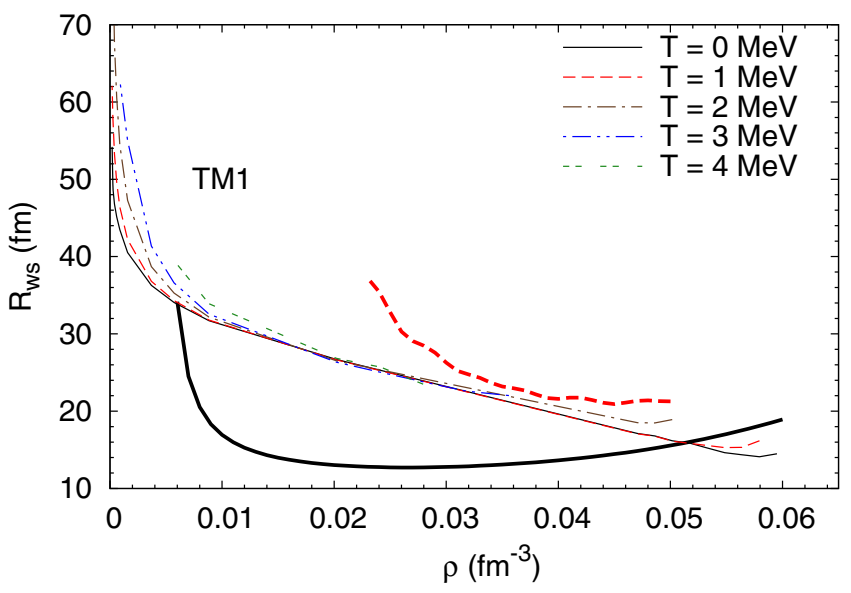

FIG. 8. (Color online) Radius of the cells as a function of matter density for the TM1 model within the DS calculation (thick lines) and TF calculation (thin lines). behavior was obtained within a different formalism; namely, considering plane-wave density fluctuations and relating the wave number of the unstable modes with the Wigner-Seitz cell size. In Refs. [27] and [26], both within the framework of the nonrelativistic Skyrme interactions and the RMF nuclear models, the Wigner-Seitz cell increases with the temperature. In this case the size of the Wigner-Seitz cell is identified to the wavelength of the perturbation, and the cluster size corresponds to half wavelength, and the system does not have the freedom to have the size of the cluster and of the cell uncorrelated.

We have performed a calculation of the WS radius within a DS calculation for TM1 and $T=0$, and $1 \mathrm{MeV}$ (thick lines in Fig. 8). TF and DS give similar sizes for $T=0 \mathrm{MeV}$ at the bottom of the inner crust, where the dynamical calculation predicts nonhomogeneous matter. However, in general the DS predictions are quite different from the TF results; in particular; quite smaller at $T=0 \mathrm{MeV}$ and larger at finite $T$.

\section{CONCLUSIONS}

In the present work the inner crust, including the nonspherical pasta phases, was calculated within a self-consistent Thomas-Fermi approach [7,8] for $\beta$-equilibrium matter at zero and finite temperature. Several relativistic nuclear models, both with constant and density-dependent couplings, were considered.

All models, except NL3 and TM1, both with a symmetry energy slope at saturation above $110 \mathrm{MeV}$, predict the existence of lasagna-like structures that may have an important contribution to the specific heat of the crust [48].

The effect of the inner crust EoS on the neutron star profiles was also analyzed. It was verified that a smaller slope gives rise to a steeper crust density profile and a larger inner crust with respect to the total crust. It may also enhance the slab phase size as observed in IU-FSU.

It was observed that the star profile obtained by using the TF inner-crust calculation or the homogeneous EoS above the outer-inner-crust transition did not differ much except for the models with a large symmetry-energy slope.

The finite-temperature calculation of the pasta phase in $\beta$ equilibrium has shown that nonhomogeneous matter is expected for temperatures below 5 to $6 \mathrm{MeV}$; the only exception was obtained with the parametrization IU-FSU which only melts at $9.5 \mathrm{MeV}$. Nonspherical structures, rod like and slab like, melt above 2 to $3.5 \mathrm{MeV}$ and 1 to $3 \mathrm{MeV}$, respectively. It was also verified that the onset density of the rod-like and the slab-like structures is independent of temperature.

Finally, it was shown that a DS calculation gives a good prediction of the crust-core transition, even at finite temperature. However, considering the size of the WS cells, this formalism fails except for $T=0 \mathrm{MeV}$ close to the crust-core transition.

\section{ACKNOWLEDGMENTS}

We are grateful to Silvia Chiacchiera for the careful reading of the manuscript and for editing the figures. This 
work was partially supported by QREN/FEDER, the Programme COMPETE, and FCT (Portugal) under the projects PTDC/FIS/113292/2009 and CERN/FP/123608/2011, by the Capes/FCT No. 232/09 bilateral collaboration and by NewCompStar, a COST initiative. H.P. is supported by FCT under Project No. SFRH/BPD/95566/2013.
[1] D. G. Ravenhall, C. J. Pethick, and J. R. Wilson, Phys. Rev. Lett. 50, 2066 (1983).

[2] M. Hashimoto, H. Seki, and M. Yamada, Prog. Theor. Phys. 71, 320 (1984).

[3] C. J. Horowitz, M. A. Pérez-Garcia, and J. Piekarewicz, Phys. Rev. C 69, 045804 (2004).

[4] G. Watanabe, K. Sato, K. Yasuoka, and T. Ebisuzaki, Phys. Rev. C 66, 012801 (2002); 68, 035806 (2003); 69, 055805 (2004); H. Sonoda, G. Watanabe, K. Sato, K. Yasuoka, and T. Ebisuzaki, ibid. 77, 035806 (2008).

[5] F. Douchin and P. Haensel, A\&A 380, 151 (2001).

[6] T. Maruyama, T. Tatsumi, D. N. Voskresensky, T. Tanigawa, and S. Chiba, Phys. Rev. C 72, 015802 (2005).

[7] S. S. Avancini, D. P. Menezes, M. D. Alloy, J. R. Marinelli, M. M. W. Moraes, and C. Providência, Phys. Rev. C 78, 015802 (2008).

[8] S. S. Avancini, L. Brito, J. R. Marinelli, D. P. Menezes, M. M. W. de Moraes, C. Providência, and A. M. Santos, Phys. Rev. C 79, 035804 (2009).

[9] S. S. Avancini, S. Chiacchiera, D. P. Menezes, and C. Providência, Phys. Rev. C 82, 055807 (2010); 85, 059904(E) (2012).

[10] H. Pais and J. R. Stone, Phys. Rev. Lett. 109, 151101 (2012).

[11] K. Oyamatsu and K. Iida, Phys. Rev. C 75, 015801 (2007).

[12] F. Grill, C. Providência, and S. S. Avancini, Phys. Rev. C 85, 055808 (2012).

[13] S. S. Avancini, C. C. Barros, Jr., L. Brito, S. Chiacchiera, D. P. Menezes, and C. Providência, Phys. Rev. C 85, 035806 (2012).

[14] C. Providência et al., Eur. Phys. J. A 50, 44 (2014).

[15] D. Page and S. Reddy, Phys. Rev. Lett. 111, 241102 (2013).

[16] B. Link, R. I. Epstein, and J. M. Lattimer, Phys. Rev. Lett. 83, 3362 (1999).

[17] N. Andersson, K. Glampedakis, W. C. G. Ho, and C. M. Espinoza, Phys. Rev. Lett. 109, 241103 (2012).

[18] J. Piekarewicz, F. J. Fattoyev, and C. J. Horowitz, Phys. Rev. C 90, 015803 (2014).

[19] W. Steiner, Stefano Gandolfi, Farrukh J. Fattoyev, and William G. Newton, arXiv:1403.7546.

[20] C. J. Horowitz, M. A. Perez-Garcia, D. K. Berry, and J. Piekarewicz, Phys. Rev. C 72, 035801 (2005).

[21] H. Sonoda, G. Watanabe, K. Sato, T. Takiwaki, K. Yasuoka, and T. Ebisuzaki, Phys. Rev. C 75, 042801 (2007).

[22] J. Margueron, J. Navarro, and N. Van Giai, Phys. Rev. C 66, 014303 (2002); F. Gulminelli, Ad. R. Raduta, M. Oertel, and J. Margueron, ibid. 87, 055809 (2013).

[23] J. R. Oppenheimer and G. M. Volkoff, Phys. Rev. 55, 374 (1939); R. C. Tolman, ibid. 55, 364 (1939).

[24] C. Providência, L. Brito, S. S. Avancini, D. P. Menezes, and Ph. Chomaz, Phys. Rev. C 73, 025805 (2006).
[25] L. Brito, C. Providência, A. M. Santos, S. S. Avancini, D. P. Menezes, and Ph. Chomaz, Phys. Rev. C 74, 045801 (2006).

[26] H. Pais, A. Santos, L. Brito, and C. Providência, Phys. Rev. C 82, 025801 (2010); H. Pais, A. Santos, and C. Providência, ibid. 80, 045808 (2009).

[27] C. Ducoin, C. Providência, A. M. Santos, L. Brito and Ph. Chomaz, Phys. Rev. C 78, 055801 (2008).

[28] C. J. Pethick and A. Y. Potekhin, Phys. Lett. B 427, 7 (1998).

[29] G. Watanabe, K. Iida, and K. Sato, Nucl. Phys. A 676, 455 (2000); 687, 512 (2001); 726, 357 (2003).

[30] J. P. Bondorf, R. Donangelo, I. N. Mishustin, C. J. Pethick, H. Schulz, and K. Sneppen, Nucl. Phys. A 443, 321 (1985); J. Bondorf, R. Donangelo, I. N. Mishustin, and H. Schulz, ibid. 444, 460 (1985); H. W. Barz, J. P. Bondorf, R. Donangelo, I. N. Mishustin, and H. Schulz, ibid. 448, 753 (1986).

[31] J. Boguta and A. R. Bodmer, Nucl. Phys. A 292, 413 (1977).

[32] S. Typel and H. H. Wolter, Nucl. Phys. A 656, 331 (1999).

[33] G. A. Lalazissis, J. König, and P. Ring, Phys. Rev. C 55, 540 (1997).

[34] Y. Sugahara and H. Toki, Nucl. Phys. A 579, 557 (1994).

[35] C. J. Horowitz and J. Piekarewicz, Phys. Rev. Lett. 86, 5647 (2001); Phys. Rev. C 64, 062802 (2001).

[36] B. G. Todd-Rutel and J. Piekarewicz, Phys. Rev. Lett. 95, 122501 (2005)

[37] F. J. Fattoyev, C. J. Horowitz, J. Piekarewicz, and G. Shen, Phys. Rev. C 82, 055803 (2010).

[38] L. Bonanno and A. Sedrakian, Astron. Astrophys. 539, A16 (2012).

[39] F. J. Fattoyev and J. Piekarewicz, Phys. Rev. C 82, 025810 (2010).

[40] G. A. Lalazissis, T. Niksić, D. Vretenar, and P. Ring, Phys. Rev. C 71, 024312 (2005).

[41] X. Roca-Maza, X. Viñas, M. Centelles, P. Ring, and P. Schuck, Phys. Rev. C 84, 054309 (2011).

[42] J. N. Negele and D. Vautherin, Nucl. Phys. A 207, 298 (1973).

[43] F. Grill, J. Margueron, and N. Sandulescu, Phys. Rev. C 84, 065801 (2011).

[44] G. Baym, C. Pethick, and P. Sutherland, Astrophys. J. 170, 299 (1971).

[45] M. Gearheart, W. G. Newton, J. Hooker, and Bao-An Li, Mon. Not. R. Astron. Soc. 418, 2343 (2011).

[46] W. G. Newton, M. Gearheart, and B.-A. Li, Astrophys. J., Suppl. Ser. 204, 9 (2013).

[47] C. Ducoin, J. Margueron, and C. Providência, Europhys. Lett. 91, 32001 (2010); C. Ducoin, J. Margueron, C. Providência, and I. Vidaña, Phys. Rev. C 83, 045810 (2011).

[48] L. Di Gallo, M. Oertel, and M. Urban, Phys. Rev. C 84, 045801 (2011). 